\section{The Role of Intention-to-Treat Analyses in Randomized Trials}

To the Editor-Fritz et al $^{1}$ recently examined 4 different approaches to eradicating Staphylococcus aureus carriage among patients with community-onset skin and soft-tissue infections (SSTIs). The increasing incidence of SSTIs-especially in the context of community-associated methicillin-resistant $S$. aureus -and the relative paucity of data on this subject make this an important investigation. The authors undertook an ambitious and rigorous approach to answering their research question, ultimately producing high-quality evidence supporting the use of dilute bleach bath, intranasal mupirocin, and hygiene education to eradicate carriage of $S$. aureus.

In the course of their analysis, the authors describe using a "modified intention-to-treat analysis" to assess their outcomes. A recent investigation by Abraha and Montedori ${ }^{2}$ examined the use of the term "modified intention to treat" (ITT) in 475 publications of randomized and controlled trials; their conclusions revealed that significant variation exists in its definition, making its meaning nearly indiscernible. In the study by Fritz et al, the authors used the term to refer to their primary analysis, which included only subjects completing follow-up visits and may more commonly be characterized as a "perprotocol" or "as-treated" analysis. While it is easy to follow the methods the authors used in their modified ITT analysis, we feel use of this term should be avoided.

One assessment not performed in this investigation was a strict ITT analysis, which treats subjects lost to follow-up as having experienced treatment failure. On the basis of information included in their publication, Table 1 presents the results from a strict ITT analysis alongside the results reported in Table 2 of the original publication. Although the original results presented in the article showed the combination of education, intranasal mupirocin, and bleach baths to be most effective at eradicating carriage of $S$. aureus, this group also had the highest attrition. In the strict ITT analysis, the advantage in this group disappears, and the eradication rate of $S$. aureus carriage is comparable to that of the control group as well as the other treatment groups.

Both strict ITT and as-treated analyses should be used to inform the overall conclusions of any given randomized trial. Both analyses provide estimates of the true effect-which likely lies somewhere between the 2 estimates-while offering different trade-offs. Strict ITT analyses ensure that the benefits of randomization, most notably the equal distribution of unmeasured confounders, remain intact. However, by treating those lost to follow-up as having experienced treatment failure, strict ITT analyses may be overly conservative and likely dilute true effect sizes. As-treated analyses may more accurately represent the impact of a given treatment by including only those who actually received treatment in the analysis. The trade-off here is that selection bias may be introduced because of differential loss to follow-up if subjects are lost due to an unmeasured or unknown confounder.

We applaud the work of these investigators and look forward to reading further work from them on this topic. We feel it necessary that clinicians are aware of the nuances in reporting clinical trials and the important role ITT analysis plays in assessing the results of randomized controlled trials.

\section{ACKNOWLEDGMENTS}

Potential conflicts of interest. All authors report no conflicts of interest relevant to this article. All authors submitted the ICMJE Form for Disclosure of Potential Conflicts of Interest, and the conflicts that the editors consider relevant to this article are disclosed here.

Joshua C. Herigon, MPH; Jason G. Newland, MD $^{1,2}$

TA BLE 1. Comparison of Strict Intention-to-Treat (ITT) and Modified ITT Analyses of Data from Fritz et al ${ }^{l}$

\begin{tabular}{|c|c|c|c|c|c|c|c|c|}
\hline & \multicolumn{2}{|c|}{ Hygiene education only } & \multicolumn{2}{|c|}{ Education and mupirocin } & \multicolumn{2}{|c|}{$\begin{array}{l}\text { Education, mupirocin, } \\
\text { and chlorhexidine }\end{array}$} & \multicolumn{2}{|c|}{$\begin{array}{l}\text { Education, mupirocin, } \\
\text { and bleach baths }\end{array}$} \\
\hline & Strict ITT & Modified ITT & Strict ITT & Modified ITT & Strict ITT & Modified ITT & Strict ITT & Modified ITT \\
\hline \multicolumn{9}{|l|}{1 month } \\
\hline Proportion (\%) & $24 / 75(32)$ & $24 / 64(38)$ & $35 / 75(47)$ & $35 / 62(56)^{a}$. & $35 / 75(47)$ & $35 / 64(55)$ & $34 / 75(45)$ & $34 / 54(63)^{2}$ \\
\hline$P$ value & $\mathrm{NA}$ & NA & .066 & .03 & .066 & .05 & .094 & .006 \\
\hline $\mathrm{RR}$ & NA & NA & 1.46 & 1.51 & 1.46 & 1.46 & 1.42 & 1.68 \\
\hline $95 \% \mathrm{CI}$ & NA & $\mathrm{NA}$ & $0.97-2.12$ & $1.02-2.21$ & $0.97-2.12$ & $0.99-2.15$ & $0.94-2.14$ & $1.15-2.44$ \\
\hline \multicolumn{9}{|l|}{4 months } \\
\hline Proportion (\%) & $31 / 75(41)$ & $31 / 64(48)$ & $32 / 75(43)$ & $32 / 57(56)$ & $31 / 75(41)$ & $31 / 57(54)$ & $36 / 75(48)$ & $36 / 51(71)^{\mathrm{a}}$ \\
\hline$P$ value & $\mathrm{NA}$ & $\mathrm{NA}$ & .869 & .40 & $>.999$ & .51 & .412 & .02 \\
\hline $\mathrm{RR}$ & NA & NA & 1.03 & 1.16 & 1.00 & 1.12 & 1.16 & 1.46 \\
\hline $95 \% \mathrm{CI}$ & NA & NA & $0.71-1.50$ & $0.82-1.63$ & $0.68-1.46$ & $0.79-1.58$ & $0.81-1.66$ & $1.07-1.98$ \\
\hline
\end{tabular}

Nоте. The values used under the heading "Modified ITT" are taken verbatim from the original investigation; these were not recalculated in our analysis. CI, confidence interval; NA, not applicable (indicates the comparator group); RR, risk ratio.

a Statistically significant difference compared with the hygiene-education-only group. 
Affiliations: 1. Children's Mercy Hospital and Clinics, Section of Infectious Diseases, University of Missouri-Kansas City School of Medicine, Kansas City, Missouri; 2. Department of Pediatrics, Section of Infectious Diseases, University of Missouri-Kansas City School of Medicine, Kansas City, Missouri.

Address correspondence to Jason G. Newland, MD, Children's Mercy Hospital and Clinics, Section of Infectious Diseases, 2401 Gillham Road, Kansas City, MO (jnewland1@cmh.edu).

Infect Control Hosp Epidemiol 2012;33(2):207-208

(C) 2012 by The Society for Healthcare Epidemiology of America. All rights reserved. 0899-823X/2012/3302-0019\$15.00. DOI: $10.1086 / 663963$

\section{REFERENCES}

1. Fritz SA, Camins BC, Eisenstein KA, et al. Effectiveness of measures to eradicate Staphylococcus aureus carriage in patients with community-associated skin and soft-tissue infections: a randomized trial. Infect Control Hosp Epidemiol 2011;32:872-880.

2. Abraha I, Montedori A. Modified intention to treat reporting in randomised controlled trials: systematic review. BMJ 2010;340: c2697.

\section{Reply to Herigon and Newland}

To the Editor-We appreciate the interest of Herigon and Newland ${ }^{1}$ in our trial of Staphylococcus aureus decolonization measures in patients with community-associated skin and soft-tissue infections. ${ }^{2}$ These authors raise an important issue in the reporting of randomized controlled trials that has been a source of much debate and has received considerable attention: handling missing outcomes in intention-to-treat (ITT) analyses.

The lack of a clear definition for the term "intention to treat" has resulted in inconsistencies in the reporting of clinical trials. ${ }^{3,4}$ The Cochrane Handbook for Systematic Reviews of Interventions ${ }^{5}$ describes 3 principles of ITT analysis, although the application of all criteria is not clearly agreed upon: (1) analyzing participants in their randomized intervention group, regardless of whether the assigned intervention actually occurred (which is generally accepted); (2) measuring outcome data for all participants (which is nearly impossible); and (3) analyzing all randomized participants (which may involve imputing data for participants with missing outcomes). Some trials use other analytic methods, including "per-protocol" analysis, which includes only participants who were known to comply with the allocated intervention and who completed the trial, and "treatmentreceived" or "as-treated" analysis, in which participants, regardless of their randomization assignment, are analyzed by the intervention that was performed. ${ }^{5}$

As earlier CONSORT (Consolidated Standards of Reporting Trials) guidelines ${ }^{6}$ recommended the use of ITT analysis when analyzing randomized trial data, the term "modified ITT analysis" is now being utilized with increasing frequency to reflect missing outcome data or protocol deviations. ${ }^{7}$ As
Herigon and Newland (and others) point out, the meaning of the term "modified ITT" is not uniformly applied. ${ }^{5,7}$ Indeed, a more accurate definition of the analyses performed in our trial is "available case analysis," in which only participants with outcome data available at longitudinal study visits were included and participants were analyzed in the arm to which they were assigned, regardless of compliance with the assigned regimen. ${ }^{5,7}$ Of note, the revised CONSORT 2010 statement requests that trial reports include whether the analysis was conducted by retaining participants in their originally assigned groups, replacing the prior guidance to report whether an "intention-to-treat" analysis was conducted.

As no consensus exists for handling missing data in ITT analyses, clinical trial experts recommend designing and conducting studies in a manner that minimizes losses to followup. ${ }^{3,4}$ Our patient population had a high prevalence of predictors of attrition reported in prior studies; ${ }^{9} 10 \%$ of our study participants reported not having a permanent home, and $15 \%$ and $51 \%$ reported having no health insurance or public health insurance, respectively. Strategies to maximize retention included a 2-staged enrollment process, flexible scheduling, cash remuneration for time and travel, and obtaining multiple phone numbers and contact information for people close to participants. ${ }^{9}$

Missing data in clinical trials is largely inevitable. However, the interpretation of missing outcome values is controversial and can be addressed in several ways. One method is to impute values for the missing data, assuming that all participants lost to follow-up experienced the event or did not experience the event. ${ }^{5}$ Herigon and Newland examined our data with one extreme assumption: that all participants lost to follow-up remained colonized with $S$. aureus. Analyzing the data with the opposite assumption, in which all participants with missing data were eradicated of $S$. aureus colonization, supports our original findings determined by available case analysis (Table 1). However, as suggested by Herigon and Newland, imputation of missing values with either the best or the worst case value results in biased results and is often too extreme. ${ }^{10}$ Another method for imputation of missing values is "last observation carried forward" (LOCF). Of note, a patient's colonization status after any intervention is confounded by multiple factors (eg, exposure to other colonized household members, interval antibiotics), and spontaneous decolonization without intervention may occur in up to $50 \%$ of participants. ${ }^{11}$ In addition, colonization was a requirement for study enrollment. Thus, we believe that the LOCF method would introduce additional bias into our study (Table 1). Ultimately, statistical techniques cannot adequately compensate for missing values. ${ }^{5}$ The missing data in our study are considered "missing completely at random" (MCAR), and the available case analysis approach is a valid interpretation of outcomes data if the MCAR assumption is met. ${ }^{5,10}$ Logistic regression analysis, including demographic and epidemiologic factors, was performed to detect significant differences between patients with and without missing longitudinal data. 